\title{
Asymptotic Time Averages and Frequency Distributions
}

\author{
Muhammad El-Taha \\ Department of Mathematics and Statistics, University of Southern Maine, 96 Falmouth Street, \\ Portland, ME 04104-9300, USA \\ Correspondence should be addressed to Muhammad El-Taha; el-taha@maine.edu
}

Received 7 March 2016; Accepted 17 July 2016

Academic Editor: MJ Lopez-Herrero

Copyright (C) 2016 Muhammad El-Taha. This is an open access article distributed under the Creative Commons Attribution License, which permits unrestricted use, distribution, and reproduction in any medium, provided the original work is properly cited.

Consider an arbitrary nonnegative deterministic process (in a stochastic setting $\{X(t), t \geq 0\}$ is a fixed realization, i.e., sample-path of the underlying stochastic process) with state space $S=(-\infty, \infty)$. Using a sample-path approach, we give necessary and sufficient conditions for the long-run time average of a measurable function of process to be equal to the expectation taken with respect to the same measurable function of its long-run frequency distribution. The results are further extended to allow unrestricted parameter (time) space. Examples are provided to show that our condition is not superfluous and that it is weaker than uniform integrability. The case of discrete-time processes is also considered. The relationship to previously known sufficient conditions, usually given in stochastic settings, will also be discussed. Our approach is applied to regenerative processes and an extension of a well-known result is given. For researchers interested in sample-path analysis, our results will give them the choice to work with the time average of a process or its frequency distribution function and go back and forth between the two under a mild condition.

\section{Introduction}

In this article we seek weak conditions, necessary and sufficient, for the long-run time average of a process or any measurable function of it to be equal to the expectation taken with respect to its long-run frequency distribution. Throughout the paper we use a sample-path approach (see [1-3]) in the sense that we restrict attention to one realization (sample-path) of the process of interest. Our approach reveals that the stochastic assumptions (regenerative, semistationary, etc.) in the most part are not needed for this result to hold, but rather to ensure that the process itself is ergodic and that a stationary distribution exists. In other words, the longrun time averages for different sample-paths of the given stochastic process converge to a common limiting value with probability one (see Example 17 in Section 4). Our approach is intuitive and the proofs are rather elementary; nothing beyond Riemann-Stieltjes integration theory is needed.

Let $\{X(t), t \geq 0\}$ be an arbitrary real-valued rightcontinuous deterministic process (in a stochastic setting
$\{X(t), t \geq 0\}$ can be a fixed realization, that is, samplepath of the underlying stochastic process) with state space $S=(-\infty, \infty)$. Let

$$
\begin{aligned}
\bar{X}_{t} & =t^{-1} \int_{0}^{t} X(u) d u, \quad t>0 ; \\
\bar{Y}_{t} & =t^{-1} \int_{0}^{t} h(X(u)) d u, \quad t>0 ; \\
G_{t}(x) & =t^{-1} \int_{0}^{t} 1\{X(u) \leq x\} d u, \\
& t>0, x \in(-\infty, \infty),
\end{aligned}
$$

where $\mathbf{1}\{\}$ is an indicator function and $h$ is a real-valued measurable function. It is also assumed that $h$ is integrable with respect to $G(x)$ in the Riemann-Stieltjes sense. Define the following limits when they exist: 


$$
\begin{aligned}
\bar{X} & =\lim _{t \rightarrow \infty} \bar{X}_{t} ; \\
\bar{Y} & =\lim _{t \rightarrow \infty} \bar{Y}_{t} ; \\
G(x) & =\lim _{t \rightarrow \infty} G_{t}(x) .
\end{aligned}
$$

Let $\bar{G}_{t}(x)$ and $\bar{G}(x)$ be the complements of $G_{t}(x)$ and $G(x)$, respectively (it follows that $\bar{G}(x)=\lim _{t \rightarrow \infty} \bar{G}_{t}(x)$ ).

Note that $\bar{X}$ and $\bar{Y}$ are the long-run time average of the processes $\{X(t), t \geq 0\}$ and $\{h(X(t)), t \geq 0\}$, respectively, and $G(x)$ is the long-run frequency distribution of $\{X(t), t \geq$ $0\}$. In a queueing system $\bar{X}$ may represent the long-run average number of customers in the system, $L$, and $G(x)$ is the "stationary distribution."

At an elementary level the problem can be posed as follows. It is of interest to establish conditions under which the long-run time average of a given process is equal to the expectation taken with respect to its long-run frequency distribution; that is, the following relation holds:

$$
\bar{X}=\int_{-\infty}^{\infty} x d G(x) .
$$

It turns out that in our sample-path setting, when $-\infty<\bar{X}<$ $+\infty$, there is a necessary and sufficient condition for relation (3) to hold. Relation (3) may also be valid even when $\bar{X}=$ $\pm \infty$.

In a stochastic setting relation (3) may have the following interpretation: for each $t>0$, let $\left\{X_{R}(t), t \geq 0\right\}$ be a process such that, for each $t, X_{R}(t)$ has $G_{t}(x)$ as its distribution function. Then the process $\left\{X_{R}(t), t \geq 0\right\}$ represents the status of the original process $\{X(t), t \geq 0\}$ as seen by a random observer that arrives at a random time uniformly distributed between 0 and $t$. If we let $X_{R}$ be a random variable with $G(x)$ as its distribution function, then $X_{R}$ describes the behavior of the process $\left\{X_{R}(t), t \geq 0\right\}$ in steady state, and relation (3) may be written as $\bar{X}=E X_{R}$, where $E X_{R}$ is the expected value of $X_{R}$.

This problem has theoretical as well as practical significance. For example, in queueing theory the long-run average number of customers in a queueing system is, sometimes, defined as $L=\lim _{t \rightarrow \infty} \int_{0}^{t} L(u) d u / t$, where $\{L(t), t \geq 0\}$ represents the number of customers present in the system (both in queue and service) at instant $t$. However, in applications, $L$ is usually calculated as the expectation of the stationary distribution $\left\{p_{n}\right\}$, of the process $\{L(t), t \geq 0\}$, provided it exists; that is, $L=\sum_{n=0}^{\infty} n p_{n}$. The question arises as to when the above two quantities are equal, particularly when $\{L(t), t \geq$ $0\}$ is not stationary and ergodic. Similar questions arise also when calculating other performance measures for queueing systems such as $W$, the long-run average waiting time in the system per customer. Another example arises when dealing with the ASTA (Arrivals that See Time Averages) property (see [1,4-8]). Following $[4,5]$ the ASTA problem is posed as follows: Given two stochastic processes $X \equiv$ $\{X(t), t \geq 0\}$ and $N \equiv\{N(t), t \geq 0\}$ defined on a common probability space, where $X$ is intended to represent a queue or a network of queues, and $N$ as an arrival point process, let
$T_{n}=\inf \{t: N(t) \geq n\}, n \geq 1$, be an imbedded point process, $X(t) \Rightarrow X(\infty)$, and $X\left(T_{n}\right) \Rightarrow \widetilde{X}(\infty)$, where $\Rightarrow$ denotes weak convergence [9]. Then the ASTA problem is to find conditions under which $X(\infty) \stackrel{d}{=} \widetilde{X}(\infty)$, where $\stackrel{d}{=}$ denotes equality in distribution. However, rather than working to verify $X(\infty) \stackrel{d}{=}$ $\widetilde{X}(\infty)$ directly, Melamed and Whitt $[4,5]$ find conditions such that $V(\infty)=W(\infty)$, where

$$
\begin{gathered}
V(\infty)=\lim _{t \rightarrow \infty} t^{-1} \int_{0}^{t} h(X(u)) d u, \\
W(\infty)=\lim _{t \rightarrow \infty} \frac{1}{N(t)} \sum_{k=1}^{N(t)} h\left(\mathrm{X}\left(T_{k}\right)\right),
\end{gathered}
$$

and $h$ is a bounded measurable real-valued function. In general $V(\infty)$ and $E h(X(\infty))$ (equivalently $W(\infty)$ and $E h(\widetilde{X}(\infty)))$ are not necessarily equal unless $X$ is stationary and ergodic. On the other hand, Stidham Jr. and El-Taha [1] prove ASTA by working directly with sample-path versions of $V(\infty)$ and $W(\infty)$. In this paper we establish weak conditions (necessary and sufficient) to guarantee the equality of time averages of a process and the expectation taken with respect to its long-run frequency distribution without explicitly assuming stationarity and ergodicity.

In a stochastic framework this problem has been treated by many authors, mostly to establish conditions that guarantee ergodicity. For example, the case of a process with an imbedded stationary sequence (the semistationary process) is proved by $[10,11]$. Franken et al. [12] derive similar results for processes with imbedded marked point processes. Rolski [13] introduces and exploits the notion of ergodically stable processes to prove a similar result. Wolff [14] proves a similar result for processes that are regenerative or nonnegative and stochastically increasing. The case for stochastic clearing processes is considered by [15]. Relevant also are [16-20]. For references on sample-path analysis, the reader is referred to $[1,2,16,21-25]$. This article should be of interest to readers with interest in establishing relationships that involve time averages and frequency distributions in a deterministic framework.

El-Taha and Stidham Jr. [2] provide a result in a samplepath setting for the special case of a nonnegative deterministic process. In this article the results of [2] are extended to any function of the process; thus all moments of a process can be treated within one framework. We also extend the state space to allow for the process to take negative as well as positive values. Moreover we consider the case where the parameter space is extended from $[0, \infty)$ to $(-\infty, \infty)$.

The article is organized as follows. In Section 2 we concentrate on the case when $\{X(t), t \geq 0\}$ is a general unrestricted process and give necessary and sufficient conditions (see conditions $A 1$ and $A 2$ in Section 2) when a measurable function, satisfying a weak regularity condition, of the process is considered. We also provide some insight into the relationship between our condition and uniform integrability. We also extend our results to allow the parameter $t$ to be unrestricted in sign. Section 3 provides similar results when $\mathbf{X}$ is a discrete-time process. In Section 4 three examples are 
given to help clarify the need for condition $A 1$, the relation of condition $A 1$ to uniform integrability, and the relationship between our pure sample-path setting and stochastic settings. In Section 5 we close by looking at regenerative processes. A process with an imbedded sequence is investigated and then used to extend a well-known result for regenerative processes.

\section{Main Results and Related Analysis}

In this section we prove the main result, provide several applications, study the connection of the conditions needed to uniform integrability, and point out an extension of the parameter space to allow negative time. Given any deterministic process, we show that for any measurable function of the process the asymptotic time average of a function of a given process is equal to the expectation taken with respect to its asymptotic frequency distribution function under weak conditions. Then several special cases of interest will be stated. Our objective is to seek weak conditions under which the asymptotic time average of a function of a given process is equal to the expectation taken with respect to it asymptotic frequency distribution function; that is,

$$
\lim _{t \rightarrow \infty} t^{-1} \int_{0}^{t} h(X(u)) d u=\int_{-\infty}^{\infty} h(x) d G(x) .
$$

Note that (5) reduces to (3) when $h$ is an identity function. First we establish the following time dependent relationships in Lemmas 1-3, key to proving the main result.

Lemma 1. Let $h(x)$ be any real-valued measurable function and $G_{t}(x)$ a distribution function defined on $(-\infty,+\infty)$. Then for all $\alpha \geq 0, t>0$

$$
\int_{\alpha}^{\infty} h(x) d G_{t}(x)=\int_{\alpha}^{\infty} \bar{G}_{t}(x) d h(x)+h(\alpha) \bar{G}_{t}(\alpha),
$$

(ii)

$$
\begin{aligned}
& \int_{-\infty}^{-\alpha} h(x) d G_{t}(x) \\
& \quad=\int_{\alpha}^{\infty} G_{t}(-x) d h(-x)+h(-\alpha) G_{t}(-\alpha),
\end{aligned}
$$

(iii)

$$
\begin{aligned}
\int_{-\infty}^{\infty} h(x) d G_{t}(x)= & \int_{0}^{\infty} \bar{G}_{t}(x) d h(x) \\
& +\int_{0}^{\infty} G_{t}(-x) d h(-x)+h(0),
\end{aligned}
$$

provided all integrals are well defined (i.e., $h(x)$ is integrable with respect to $G_{t}(x)$ in the Riemann-Stieltjes sense).
Proof. Note that

$$
\begin{aligned}
\int_{\alpha}^{\infty} h(x) d G_{t}(x)= & \int_{\alpha}^{\infty}\left[\int_{0}^{x} d h(y)+h(0)\right] d G_{t}(x) \\
= & \int_{0}^{\alpha} \int_{\alpha}^{\infty} d G_{t}(x) d h(y) \\
& +\int_{\alpha}^{\infty} \int_{y}^{\infty} d G_{t}(x) d h(y) \\
& +h(0) \bar{G}_{t}(\alpha) \\
= & \int_{\alpha}^{\infty} \bar{G}_{t}(y) d h(y)+h(\alpha) \bar{G}_{t}(\alpha)
\end{aligned}
$$

which proves part (i). The proof of (ii) is similar. Part (iii) follows by taking $\alpha=0$ in (i) and (ii) and combining both results.

The results in Lemma 1 remain valid if we replace $G_{t}(x)$ by $G(x)$ when the limit exists. Now we give the second partial result.

Lemma 2. For all $t>0$

$$
t^{-1} \int_{0}^{t} h(X(u)) d u=\int_{-\infty}^{\infty} h(x) d G_{t}(x) .
$$

Proof. Note that

$$
\begin{aligned}
& \int_{0}^{\infty} \bar{G}_{t}(x) d h(x)=\int_{0}^{\infty} t^{-1} \int_{0}^{t} \mathbf{1}\{X(u)>x\} d u d h(x) \\
& =t^{-1} \int_{0}^{t} \int_{0}^{X(u)} \mathbf{1}\{X(u)>0\} d h(x) d u \\
& =t^{-1} \int_{0}^{t} h(X(u)) \mathbf{1}\{X(u)>0\} d u-h(0) \bar{G}_{t}(0) .
\end{aligned}
$$

Similarly

$$
\begin{aligned}
\int_{0}^{\infty} & G_{t}(-x) d h(-x) \\
= & \int_{0}^{\infty} t^{-1} \int_{0}^{t} \mathbf{1}\{X(u) \leq-x\} d u d h(-x) \\
= & t^{-1} \int_{0}^{t} h(X(u)) \mathbf{1}\{X(u) \leq 0\} d u-h(0) G_{t}(0) .
\end{aligned}
$$

Then the result follows by appealing to Lemma 1 (iii).

By taking limits, as $t \rightarrow \infty$ of both sides of Lemma 2, showing relation (5) holds is seen to be equivalent to a problem of interchanging limits and integrals. The next result is a key lemma.

Lemma 3. For all $t>0$ and $\alpha \geq 0$

$$
\begin{aligned}
& \int_{\{x:|x|>\alpha\}} h(x) d G_{t}(x) \\
& =t^{-1} \int_{0}^{t} h(X(u)) \mathbf{1}\{|X(u)|>\alpha\} d u .
\end{aligned}
$$


Proof. The result will follow if we show that

(i)

$\int_{\alpha}^{\infty} h(x) d G_{t}(x)=t^{-1} \int_{0}^{t} h(X(u)) \mathbf{1}\{X(u)>\alpha\} d u$,

(ii)

$$
\begin{aligned}
& \int_{-\infty}^{-\alpha} h(x) d G_{t}(x) \\
& \quad=t^{-1} \int_{0}^{t} h(X(u)) \mathbf{1}\{X(u)<-\alpha\} d u .
\end{aligned}
$$

Now

$$
\begin{aligned}
& \int_{\alpha}^{\infty} \bar{G}_{t}(x) d h(x)=\int_{\alpha}^{\infty} t^{-1} \int_{0}^{t} \mathbf{1}\{X(u)>x\} d u d h(x) \\
& =t^{-1} \int_{0}^{t} \int_{\alpha}^{X(u)} \mathbf{1}\{X(u)>\alpha\} d h(x) d u \\
& =t^{-1} \int_{0}^{t} h(X(u)) \mathbf{1}\{X(u)>\alpha\} d u-h(\alpha) \bar{G}_{t}(\alpha) .
\end{aligned}
$$

Therefore, using Lemma 1 (i), we obtain

$$
\int_{\alpha}^{\infty} h(x) d G_{t}(x)=t^{-1} \int_{0}^{t} h(X(u)) \mathbf{1}\{X(u)>\alpha\} d u
$$

which proves part (i). Part (ii) is proved similarly using Lemma 1 (ii).

Because Lemmas 1-3 are time dependent, they can be useful in time dependent analysis of nonstationary stochastic systems. Now we give the main result.

Theorem 4. Consider the deterministic process $\{X(t), t \geq 0\}$, with state space $S=(-\infty, \infty)$, and let $h(\cdot)$ be any real-valued measurable function. Then, the following are equivalent.

(i) Condition A1:

$$
\lim _{\alpha \rightarrow \infty} \lim _{t \rightarrow \infty} t^{-1} \int_{0}^{t} h(X(u)) \mathbf{1}\{|X(u)|>\alpha\} d u=0 .
$$

(ii) Condition A2:

$$
\lim _{\alpha \rightarrow \infty} \lim _{t \rightarrow \infty} \int_{\{x:|x|>\alpha\}} h(x) d G_{t}(x)=0 .
$$

(iii)

$$
\lim _{t \rightarrow \infty}\left[t^{-1} \int_{0}^{t} h(X(u)) d u-\int_{-\infty}^{\infty} h(x) d G(x)\right]=0 .
$$

Proof. Taking the limits as $\lim _{\alpha \rightarrow \infty} \lim _{t \rightarrow \infty}$ on both sides of Lemma 3 proves the equivalence of (i) and (ii). Now, using Lemmas 3 and 2, we write

$$
\begin{aligned}
& t^{-1} \int_{0}^{t} h(X(u)) d u-\int_{-\alpha}^{+\alpha} h(x) d G_{t}(x) \\
& \quad=\int_{\{x:|x|>\alpha\}} h(x) d G_{t}(x), \\
& t^{-1} \int_{0}^{t} h(X(u)) d u-\int_{-\alpha}^{+\alpha} h(x) d G_{t}(x) \\
& =t^{-1} \int_{0}^{t} h(X(u)) \mathbf{1}\{|X(u)|>\alpha\} d u .
\end{aligned}
$$

Suppose $A 1$ (equivalently $A 2$ ) holds. Now, taking limits as $t \rightarrow \infty$ and assuming they exist give

$$
\begin{aligned}
& \lim _{t \rightarrow \infty} t^{-1} \int_{0}^{t} h(X(u)) d u-\int_{-\alpha}^{+\alpha} h(x) d G(x) \\
& =\lim _{t \rightarrow \infty} \int_{\{x:|x|>\alpha\}} h(x) d G_{t}(x), \\
& \lim _{t \rightarrow \infty} t^{-1} \int_{0}^{t} h(X(u)) d u-\int_{-\alpha}^{+\alpha} h(x) d G(x) \\
& =\lim _{t \rightarrow \infty} t^{-1} \int_{0}^{t} h(X(u)) \mathbf{1}\{|X(u)|>\alpha\} d u .
\end{aligned}
$$

Then part (iii) of the theorem follows by taking limits of both sides in (22) and (23) as $\alpha \rightarrow \infty$. Conversely if part (iii) holds, it is straightforward to see that $A 1$ and $A 2$ hold.

It follows from Theorem 4 that the following conditions are sufficient for (5) to hold.

Corollary 5. Consider the process $\{X(t), t \geq 0\}$. Suppose $G_{t}(x) \rightarrow G(x)$ uniformly in $x$ as $t \rightarrow \infty$. Suppose also that condition (i) (equivalently (ii)) of Theorem 4 holds. Then for any measurable real-valued function $h, \lim _{t \rightarrow \infty} t^{-1} \int_{0}^{t} h(X(u)) d u$ is well defined if and only if $\int_{-\infty}^{\infty} h(x) d G(x)$ is well defined, in which case relation (5) holds; that is,

$$
\lim _{t \rightarrow \infty} \frac{1}{t} \int_{0}^{t} h(X(u)) d u=\int_{-\infty}^{\infty} h(x) d G(x) .
$$

Proof. The proof follows by taking limits, as $\alpha \rightarrow \infty$, in (22) and (23) and using a similar argument as in Theorem 4.

Corollary 6. Consider the process $\{X(t), t \geq 0\}$ and suppose that the conditions of Corollary 5 hold. Additionally, let $h(\cdot)$ be differentiable and $h(0)=0$. Then

$$
\begin{aligned}
\lim _{t \rightarrow \infty} & \frac{1}{t} \int_{0}^{t} h(X(u)) d u \\
\quad & \int_{-\infty}^{0} h^{\prime}(x) G(x) d x+\int_{0}^{\infty} h^{\prime}(x) \bar{G}(x) d x .
\end{aligned}
$$


Proof. By Corollary 5, it suffices to show that

$$
\begin{aligned}
\int_{-\infty}^{\infty} h(x) d G(x)= & \int_{-\infty}^{0} h^{\prime}(x) G(x) d x \\
& +\int_{0}^{\infty} h^{\prime}(x) \bar{G}(x) d x .
\end{aligned}
$$

So, the result follows by noting that

$$
\int_{0}^{\infty} G(x) d h(-x)=\int_{-\infty}^{0} h^{\prime}(x) G(x) d x
$$

and using Lemma 1 (iii).

In Corollary 6, let $h(x)=x^{\beta}$, then (26) gives the $\beta$ th moment of a distribution function

$$
\begin{aligned}
\int_{-\infty}^{\infty} x^{\beta} d G_{t}(x)= & \int_{-\infty}^{0} \beta x^{\beta-1} G_{t}(x) d x \\
& +\int_{0}^{\infty} \beta x^{\beta-1} \bar{G}_{t}(x) d x
\end{aligned}
$$

which is familiar when $G(x)$ is defined only on $[0, \infty)$.

Remarks 7. (i) It follows immediately from Corollary 5 that when $-\infty<\bar{Y}<+\infty$ (recall $Y(t):=h(X(t)))$ condition $A 1$ (equivalently $A 2$ ) is necessary and sufficient for relation (5) to hold. The requirement that $-\infty<\bar{Y}<\infty$ is not needed to prove the sufficiency part of the assertion.

(ii) From (23), it is clear that if $\int_{-\alpha}^{+\alpha} h(x) d G(x)$ is well defined and finite for all $0<\alpha<\infty$, then $\bar{Y}$ is well defined iff $\lim _{t \rightarrow \infty} t^{-1} \int_{0}^{t} h(X(u)) \mathbf{1}\{X(u)>\alpha\} d u$ is well defined for all $\alpha>0$. Now take limits in (23) as $\alpha \rightarrow \infty$ to obtain

$$
\begin{aligned}
\lim _{t \rightarrow \infty} \frac{1}{t} \int_{0}^{t} h(X(u)) d u \\
=\int_{-\infty}^{\infty} h(x) d G(x) \\
\quad+\lim _{\alpha \rightarrow \infty} \lim _{t \rightarrow \infty} t^{-1} \int_{0}^{t} h(X(u)) \mathbf{1}\{|X(u)|>\alpha\} d u .
\end{aligned}
$$

If any two of the three terms in (29) are well defined and at least one of them is finite then the third term exists and Corollary 5 holds.

(iii) Assume that all the relevant limits in Corollary 5 are well defined. From (23) one concludes that, for all $\alpha \geq 0$,

$$
\begin{aligned}
\bar{Y}= & \pm \infty \\
& \quad \text { iff } \lim _{t \rightarrow \infty} t^{-1} \int_{0}^{t} h(X(u)) \mathbf{1}\{|X(u)|>\alpha\} d u= \pm \infty .
\end{aligned}
$$

Moreover, if $\bar{Y}=+\infty$ and condition $A 1$ is not satisfied, we can distinguish to possibilities

(a) $\int_{-\infty}^{\infty} x d G(x)=\infty$; then relation (5) holds; (b) $\int_{-\infty}^{\infty} x d G(x)<\infty$; then relation (5) does not hold and condition $A 1$ takes the value $+\infty$. A similar argument applies when $\bar{Y}=-\infty$. The above discussion should not imply that if condition $A 1$ does not hold it should be infinite. Example 15 given below shows that relation (5) fails with condition $A 1$ assuming a finite value.

(iv) One can construct sufficient conditions for condition $A 1$ to hold. Following an argument by Billingsley [26, page 186], one can easily see that $\lim _{t \rightarrow \infty} t^{-1} \int_{0}^{t}[h(X(u))]^{1+\epsilon} d u<$ $\infty$ is sufficient for (5) to hold.

Next we explore the relationship between condition $A 1$ and uniform integrability of the process $\left\{X_{R}(t), t \geq 0\right\}$.

Relation to Uniform Integrability. We discuss the connection between condition $A 1$ and uniform integrability when $h$ is an identity function. Condition $A 1$ of Theorem 4 requires, roughly speaking, that the area, for $0 \leq t \leq \infty$, between $\alpha$ and $X(t) \mathbf{1}\{X(t)>\alpha\}$ minus the area between $-\alpha$ and $X(t) \mathbf{1}\{X(t)<-\alpha\}$ goes to zero as $\alpha$ approaches infinity. We point out here that there is a difference between condition $A 1$ and uniform integrability (of $X_{R}(t)$ ) which requires that the above two areas add up to zero as $\alpha$ approaches infinity. Wolff [14] suggests that proving relation (5), in a stochastic setting, is equivalent to showing that the process $\left\{X_{R}(t), t \geq 0\right\}$ and $\bar{X}_{t}$ are uniformly integrable (u.i.) in $t$. Condition $A 1$ is weaker than being u.i.; it only coincides with uniform integrability of the process $\left\{X_{R}(t), t \geq 0\right\}$ when the process $\{X(t), t \geq 0\}$ is nonnegative.

To shed more light on this difference, we show that the following modified condition is the equivalent to uniform integrability.

Condition A3. $\lim _{\alpha \rightarrow \infty} \sup _{t} t^{-1} \int_{0}^{t}|X(u)| \mathbf{1}\{|X(u)|>\alpha\} d u=0$.

Note that in $A 3$ we take the absolute value of $X(t)$. In our sample-path setting the definition of uniform integrability $[26,27]$ of the process $\left\{X_{R}(t), t \geq 0\right\}$ is equivalent to

$$
\lim _{\alpha \rightarrow \infty} \sup _{t} \int_{\{x:|x|>\alpha\}}|x| d G_{t}(x)=0 .
$$

Now, using an argument similar to that used in proving Lemma 3, we obtain

$$
\begin{aligned}
& \int_{\{x:|x|>\alpha\}}|x| d G_{t}(x) \\
& =t^{-1} \int_{0}^{t}|X(u)| \mathbf{1}\{|X(u)|>\alpha\} d u .
\end{aligned}
$$

Thus condition $A 3$ and (31) are equivalent. We note that condition $A 3$ (equivalently (31)) is sufficient for relation (5) to hold. Example 16 given below shows the existence of a process that obeys relation (5), yet condition $A 3$ is not satisfied.

2.1. Moments. An important special case is when $h(x)=x^{\beta}$, $\beta>0$, that provides a relation between time averages of a process moments and the moments obtained by using its 
asymptotic distribution function. For $h(x)=x^{\beta}$, we have the following result.

Corollary 8. Let $h(x)=x^{\beta}$. Then the following are equivalent: (i)

$$
\lim _{\alpha \rightarrow \infty} \lim _{t \rightarrow \infty} t^{-1} \int_{0}^{t}[X(u)]^{\beta} \mathbf{1}\{|X(u)|>\alpha\} d u=0 ;
$$

(ii)

$$
\lim _{\alpha \rightarrow \infty} \lim _{t \rightarrow \infty} \int_{\{x:|x|>\alpha\}} x^{\beta} d G_{t}(x)=0
$$

(iii)

$$
\begin{aligned}
\lim _{t \rightarrow \infty} t^{-1} \int_{0}^{t}[X(u)]^{\beta} d u & =\int_{-\infty}^{\infty} x^{\beta} d G(x), \\
|\bar{Y}| & <\infty
\end{aligned}
$$

where $\bar{Y}=\lim _{t \rightarrow \infty} t^{-1} \int_{0}^{t}[X(u)]^{\beta} d u$.

Another important special case is when considering absolute moments, that is, when $h(x)=|x|^{\beta}$.

2.2. Extension. Here, a generalization of Theorem 4 will be considered. We allow the parameter space $[0, \infty)$ to extend to $(-\infty, \infty)$ and show that, for any measurable function of the new process, relation (5) remains valid under conditions similar to $A 1$ and $A 2$. Theorem 4 is extended to the case where the parameter can also be negative.

Let $\{X(t),-\infty<t<\infty\}$ be an arbitrary (deterministic) process with state space $S=(-\infty, \infty)$, and let

$$
\begin{aligned}
G_{t}(x)=\frac{1}{2 t} \int_{-t}^{t} 1\{X(u)<x\} d u, & \\
x & \in(-\infty, \infty), t>0 .
\end{aligned}
$$

Here we seek weak conditions under which the following relation holds:

$$
\lim _{t \rightarrow \infty} \frac{1}{2 t} \int_{-t}^{t} h(X(u)) d u=\int_{-\infty}^{\infty} h(x) d G(x) .
$$

Theorem 9. Consider the deterministic process $\{X(t),-\infty<$ $t<\infty\}, X(t) \in R$, and let $h(\cdot)$ be any real-valued measurable function. Then, the following are equivalent.

(i) Condition B1:

$$
\lim _{\alpha \rightarrow \infty} \lim _{t \rightarrow \infty} \frac{1}{2 t} \int_{-t}^{t} h(X(u)) \mathbf{1}\{|X(u)|>\alpha\} d u=0 .
$$

(ii) Condition B2:

$$
\lim _{\alpha \rightarrow \infty} \lim _{t \rightarrow \infty} \int_{\{x:|x|>\alpha\}} h(x) d G_{t}(x)=0 .
$$

(iii)

$$
\lim _{t \rightarrow \infty}\left[\frac{1}{2 t} \int_{-t}^{t} h(X(u)) d u-\int_{-\infty}^{\infty} h(x) d G_{t}(x)\right]=0 .
$$

Proof. Similar to Lemma 3 we can show that for all $t>0$ and $\alpha \geq 0$

$$
\begin{aligned}
& \int_{\{x:|x| \geq \alpha\}} h(x) d G_{t}(x) \\
& =\frac{1}{2 t} \int_{-t}^{t} h(X(u)) \mathbf{1}\{|X(u)| \geq \alpha\} d u .
\end{aligned}
$$

Now, let $\alpha=0$ in (41); then we have for all $t>0$

$$
\frac{1}{2 t} \int_{-t}^{t} h(X(u)) d u=\int_{-\infty}^{\infty} h(x) d G_{t}(x) .
$$

Now an argument similar to that of the proof of Theorem 4 will yield this result.

Note that proof of Theorem 9 follows the same lines of argument as that of Theorem 4 . Note also that conditions $B 1$ and $B 2$ are similar to conditions $A 1$ and $A 2$, but with $2 t$ in the denominator in $B 1$.

\section{Discrete-Time Processes}

In this section we consider a discrete-time process; specifically let $\left\{X_{n}, n \geq 1\right\}$ be any deterministic discrete-time process, that is, an infinite sequence of real numbers, and let $h(\cdot)$ be a real-valued measurable function. Moreover, let

$$
\begin{aligned}
\bar{X}_{n} & =\frac{1}{n} \sum_{k=1}^{n} X_{k} ; \\
G_{n}(x) & =\frac{1}{n} \sum_{k=1}^{n} 1\left\{X_{k} \leq x\right\} ; \quad x \in(-\infty, \infty) ;
\end{aligned}
$$

and define the following limits when they exist:

$$
\begin{aligned}
\bar{X} & =\lim _{n \rightarrow \infty} \bar{X}_{n}, \\
G(x) & =\lim _{n \rightarrow \infty} G_{n}(x), \quad x \in(-\infty, \infty) .
\end{aligned}
$$

Similar to the continuous time model, we have the following results.

Theorem 10. Let $\left\{X_{n}, n \geq 1\right\}$ be any discrete-time process, and let $h(\cdot)$ be any a real-valued measurable function. Then, the following are equivalent:

(i) $\lim _{\alpha \rightarrow \infty} \lim _{n \rightarrow \infty} n^{-1} \sum_{k=1}^{n} h\left(X_{k}\right) \mathbf{1}\left\{\left|X_{k}\right|>\alpha\right\}=0$;

(ii) $\lim _{\alpha \rightarrow \infty} \lim _{n \rightarrow \infty} \int_{\{x:|x|>\alpha\}} h(x) d G_{n}(x)=0$;

(iii) $\lim _{n \rightarrow \infty}\left[n^{-1} \sum_{k=1}^{n} h\left(X_{k}\right)-\int_{-\infty}^{\infty} h(x) d G(x)\right]=0$.

Note that when $h(\cdot)$ is an identity function, condition (i) of Theorem 10 is sometimes referred to in the literature as the condition for uniform integrability.

Corollary 11. Consider the process $\left\{X_{n}, n \geq 1\right\}$. Suppose $G_{n}(x) \rightarrow G(x)$ uniformly in $x$ as $n \rightarrow \infty$. Suppose also that condition (i) (equivalently (ii)) of Theorem 10 holds. Then for any measurable real-valued function 
$h, \lim _{n \rightarrow \infty}(1 / n) \sum_{k=1}^{n} h\left(X_{k}\right)$ is well defined if and only if $\int_{-\infty}^{\infty} h(x) d G(x)$ is well defined, in which case

$$
\lim _{n \rightarrow \infty} \frac{1}{n} \sum_{k=1}^{n} h\left(X_{k}\right)=\int_{-\infty}^{\infty} h(x) d G(x) .
$$

This corollary has been found useful in the literature.

Corollary 12. Let $\left\{X_{n}, n \geq 1\right\}$ be any nonnegative deterministic discrete-time process, that is, infinite sequence of nonnegative real numbers. Suppose that any of the conditions (i), (ii), and (iii) of Theorem 10 is satisfied and $G(x)$ is a proper distribution function. Now, suppose there exists $y>0$ such that if $h(\cdot)$ is a nondecreasing real-valued measurable function on $(y, \infty)$ then

$$
\lim _{x \rightarrow \infty} h(x) G^{c}(x)=0
$$

and if $h(\cdot)$ is a nonincreasing real-valued measurable function on $(-\infty,-y)$, then

$$
\lim _{x \rightarrow-\infty} h(x) G(x)=0 .
$$

Proof. Note that

$$
\begin{aligned}
\lim _{x \rightarrow \infty} h(x) G^{c}(x) & =\lim _{x \rightarrow \infty} \lim _{n \rightarrow \infty} h(x) G_{n}^{c}(x) \\
& =\lim _{x \rightarrow \infty} \lim _{n \rightarrow \infty} \int_{x}^{\infty} h(x) d G_{n}(u) \\
& \leq \lim _{x \rightarrow \infty} \lim _{n \rightarrow \infty} \int_{x}^{\infty} h(u) d G_{n}(u)=0,
\end{aligned}
$$

by condition (ii) of Theorem 10. The proof of the second statement is similar.

The results in Corollary 12 apply to the continuous time process as well. When the sequence $\left\{X_{k} ; k \geq 1\right\}$ represents, say, service times in queueing model, let $h(x)=x^{2}$ in Theorem 10 to, immediately, obtain the following useful results.

Corollary 13. Let $E X^{2}=\lim _{n \rightarrow \infty} n^{-1} \sum_{k=1}^{n} X_{k}^{2}<\infty$. Then the following are equivalent:

(i) $\lim _{\alpha \rightarrow \infty} \lim _{n \rightarrow \infty} n^{-1} \sum_{k=1}^{n} X_{k}^{2} 1\left\{X_{k}>\alpha\right\}=0$;

(ii) $\lim _{\alpha \rightarrow \infty} \lim _{n \rightarrow \infty} \int_{\alpha}^{\infty} x^{2} d G_{n}(x)=0$;

(iii) $E X^{2}=\int_{0}^{\infty} x^{2} d G(x)<\infty$.

Corollary 14. Suppose that either

(i) $E X^{2}=\int_{0}^{\infty} x^{2} d G(x)<\infty$ or

(ii) $\lim _{\alpha \rightarrow \infty} \lim _{n \rightarrow \infty} \sum_{k=1}^{n} X_{k}^{2} \mathbf{1}\left\{X_{k}>\alpha\right\}=0$. Then

$$
\lim _{x \rightarrow \infty} x^{2} G^{c}(x)=0 \text {. }
$$

Corollary 14 follows from Corollary 12 by letting $h(x)=$ $x^{2}$. This result is used in Ayesta [28].

\section{Examples and Discussion}

In this section, we give three examples. The first example shows that condition $A 1$ is not superfluous. The second example, a modification of the first one, shows that the new modified process does not satisfy the uniform integrability condition (condition $A 3$ ), yet conditions $A 1$ and $A 2$ are satisfied and therefore relation (5) holds. In the third example, we verify that when condition $A 1$ is satisfied, for a stationary nonergodic stochastic process, even though relation (5) is not satisfied in a stochastic setting, it remains valid for every individual sample-path of the process.

Example 15. Consider a process $\left\{X_{n}, n \geq 0\right\}$ in discrete time, and let the sequence $\left\{x_{n}\right\}$ represent one sample-path such that

$$
x_{n}= \begin{cases}2^{m}, & n=4^{m}+3 k 2^{m}-1 \\ & k=1,2, \ldots, 2^{m} \\ & m=0,1, \ldots \\ 0 \quad & \text { otherwise. }\end{cases}
$$

For example if $m=0$, then $x_{1}=0, x_{2}=0, x_{3}=1$; if $m=1$, then $x_{4}=\cdots=x_{8}=0, x_{9}=2, x_{10}=\cdots=x_{14}=$ $0, x_{15}=2$; if $m=2$, then $x_{16}=\cdots=x_{26}=0, x_{27}=4$, $x_{28}=\cdots=x_{38}=0, x_{39}=4, x_{40}=\cdots=x_{50}=0, x_{51}=4$, $x_{52}=\cdots=x_{62}=0, x_{63}=4$; if $m=3$, then $x_{64}=0, \ldots$, and so on. Figure 1 plots the cumulative sequence $\sum_{j=1}^{n} x_{j}$ for all $n$, showing the jumps at positive terms of $\left\{x_{n}\right\}$.

Now, consider the sum of the sequence $\left\{x_{n}\right\}$ at positive terms; then

$$
\begin{aligned}
\lim \sup \frac{1}{n} \sum x_{j} & =\frac{1}{2^{2 m}+3 \cdot k \cdot 2^{m}-1} \sum_{j=1}^{2^{2 m}+3 k \cdot 2^{m}-1} x_{j} \\
& =\frac{1+2^{2}+2^{4}+\cdots+2^{2(m-1)}+k \cdot 2^{m}}{4^{m}+3 k \cdot 2^{m}-1} \\
& =\frac{1}{3} .
\end{aligned}
$$

Now, consider the sum just before positive terms to obtain

$$
\begin{gathered}
\lim \inf \frac{1}{n} \sum x_{j}=\frac{1}{2^{2 m}+3 \cdot k \cdot 2^{m}-2} \sum_{j=1}^{2^{2 m}+3 k \cdot 2^{m}-2} x_{j} \\
=\frac{1+2^{2}+2^{4}+\cdots+2^{2(m-1)}+(k-1) \cdot 2^{m}}{4^{m}+3 k \cdot 2^{m}-2} \\
=\frac{1}{3}+\left(\frac{1}{3}\right) \frac{3 / 2^{m}-3}{2^{m}+3 k-2 / 2^{m}} \longrightarrow \frac{1}{3} \\
\text { as } m \longrightarrow \infty \text { for any fixed } k .
\end{gathered}
$$

Therefore $\liminf (1 / n) \sum_{j=1}^{n} x_{j}=1 / 3$, and hence $\lim _{n \rightarrow \infty}(1 / n) \sum_{j=1}^{n} x_{j}=1 / 3$. We also conclude, using Lemma 2, that

$$
\bar{x}=\lim _{n \rightarrow \infty} \int_{0}^{\infty} x d G_{n}(x)=\frac{1}{3} .
$$




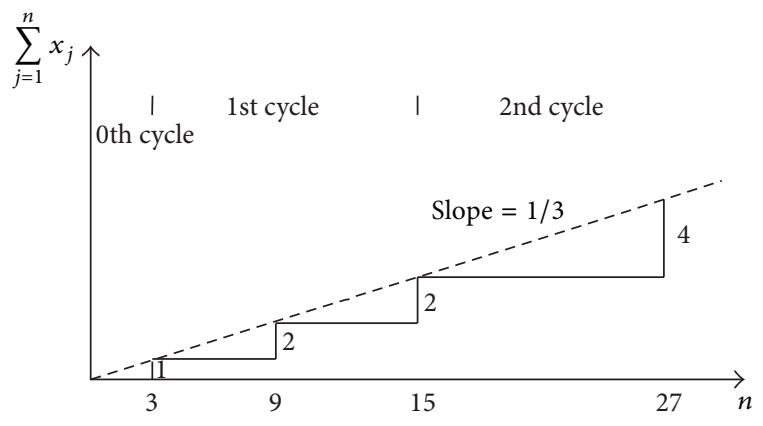

FIGURE 1: Cumulative sequence for $\left\{x_{n}\right\}$.

The next step is to calculate the r.h.s. of relation (5) and show that it differs from $1 / 3$. Now

$$
\begin{aligned}
\int_{0}^{\infty} x d G(x) & =\int_{0}^{\infty} \bar{G}(x) d x, \\
\bar{G}_{n}(x) & =\frac{1}{n} \sum_{k=1}^{n} \mathbf{1}\left\{x_{k}>x\right\} .
\end{aligned}
$$

Successive values of $m$ determine successive cycles. Now consider $\bar{G}_{n}(0)$ at the beginning of such cycles to obtain

$$
\begin{aligned}
\bar{G}_{4^{m}}(0) & =\frac{1}{4^{m}} \sum_{k=1}^{4^{m}} \mathbf{1}\left\{x_{k}>0\right\}=\frac{1+2+2^{2}+\cdots+2^{m-1}}{4^{m}} \\
& =\frac{2^{m}-1}{4^{m}} \longrightarrow 0, \quad \text { as } m \longrightarrow \infty
\end{aligned}
$$

Since $(1 / n) \sum_{k=1}^{n} \mathbf{1}\left\{x_{k}>x\right\}$ decreases in $n$ as long as $x_{n}=0$, it suffices to consider $\bar{G}_{n}(0)$ at a subsequence of positive terms. Then

$$
\begin{aligned}
\bar{G}_{4^{m}+3 k \cdot 2^{m}-1}(0) & =\frac{1+2+2^{2}+\cdots+2^{m-1}+k}{4^{m}+3 k \cdot 2^{m}-1} \\
& =\frac{2^{m}-1+k}{4^{m}+3 k 2^{m}-1} \longrightarrow 0, \\
& \text { as } m \longrightarrow \infty .
\end{aligned}
$$

Therefore $\bar{G}_{n}(x) \rightarrow 0$ as $n \rightarrow \infty$ for all $x \geq 0$ and $G_{n}(x) \rightarrow 1$ as $n \rightarrow \infty$ for all $x \geq 0$; that is, $G(x)$ is well defined and $G(x)=\lim _{n \rightarrow \infty} G_{n}(x)=1$ for all $x \geq 0$. Thus $\bar{x}=1 / 3 \neq \int_{0}^{\infty} \bar{G}(x) d x=0$, so relation (5) fails. In this example condition $A 1$ takes the value $1 / 3$.

The next example gives a sequence that does not satisfy the uniform integrability condition $A 3$, yet it satisfies relation (5).

Example 16. Now, we modify Example 15 as follows: let

$$
z_{n}=x_{n}-x_{n-1}, \quad n=1,2, \ldots
$$

with $z_{0}=0$.
Using Example 15 and the fact that $\left|z_{n}\right|=z_{n}^{+}+z_{n}^{-}$, we conclude

$$
\lim _{\alpha \rightarrow \infty} \lim _{n \rightarrow \infty} \frac{1}{n} \sum_{k=0}^{n}\left|z_{k}\right| \mathbf{1}\left\{\left|z_{k}\right|>\alpha\right\}=\frac{2}{3}
$$

thus the sequence $\left\{z_{n}\right\}$ does not satisfy the uniform integrability condition $(A 3)$. Now, using the fact that $z_{n}=z_{n}^{+}-z_{n}^{-}$, we conclude that condition $A 1$ is satisfied, and therefore relation (5) holds.

Example 17. Let the stationary stochastic process $\left\{X_{n}, n \geq 1\right\}$ be defined as

$$
\begin{aligned}
P\left\{X_{1}=k\right\} & =p_{k}, \\
E X_{1} & =\mu<\infty,
\end{aligned}
$$

where $p_{k} \geq 0, \sum_{k=0}^{\infty} p_{k}=1$, and $X_{n}=X_{1}$ for all $n \geq 1$.

The sequence $\left\{X_{n}, n \geq 1\right\}$ is an example of a stationary nonergodic process (see, e.g., [29]). The sample space is given by $S=\left\{\omega_{0}, \omega_{1}, \ldots\right\}$, where $\omega_{n}$ represents the sequence $\{n\}_{k=1}^{\infty}, n=0,1, \ldots$. It is clear that $\bar{X}\left(\omega_{n}\right)=n$ and $\int_{-\infty}^{\infty} x d G\left(x, \omega_{n}\right)=n$. It should be clear that none of the time average values $\bar{X}(w)$, except possibly one, is equal to $\mu$. So relation (5) is true for every realization of the process. On the other hand, $\bar{X}_{n}=(1 / n) \sum_{k=1}^{n} X_{k}=X_{1}$ is a random variable for all $n=1,2, \ldots$. Therefore $\bar{X}$ is a random variable, while $\int_{-\infty}^{\infty} x d G(x)=E X_{1}=\mu$; thus relation (5) does not hold with probability one.

To extend our results, that is, relation (5), to stochastic settings an ergodicity assumption is needed; more precisely, we need to insure that the time average $\bar{X}$ in relation (5) assumes a constant value, say, $m$, with probability one. To illustrate, let the event $A=\{w \in \Omega$ : relation (5) fails $\}$, then $A$ contains the following realizations: (i) sample-paths for which the limits in relation (5) do not exist; (ii) samplepaths for which the limits exist-but relation (5) does not hold (as in Example 15); (iii) sample-paths for which relation (5) holds, but $\bar{X} \neq m$.

Relation (5) holds in a stochastic setting if $P(A)=0$. In contrast, in Example 17, $P(A)=1$. Thus the following corollary follows:

Corollary 18. Let $\{X(t)\}$ be a stochastic process defined on a probability space $(\Omega, \mathscr{F}, P)$. If there exists a constant $m$ such that $\bar{X}=m$ with probability one, then (with probability one) Condition $A 2$ is satisfied iff $|\bar{X}|<\infty$, and relation (5) holds.

\section{Application to Regenerative Processes}

In this section, we give a simple proof of relation (5) for regenerative processes. Consider a general process with an imbedded point process. Here we need only to consider the limit in condition $A 1$ at the imbedded sequence of time points and then specialize to regenerative processes and extend a result by Wolff [14]. Let $\{X(t), t \geq 0\}$ be any process with 
$\left\{T_{n}\right\}, n=0,1, \ldots, T_{0}=0$, be an imbedded point process. Define

$$
\begin{aligned}
N(t) & =\max \left\{n: T_{n} \leq t\right\}, \\
\lambda & =\lim _{t \rightarrow \infty} \frac{N(t)}{t}, \quad \lambda \in(0, \infty) .
\end{aligned}
$$

Assume that $n \rightarrow \infty$ iff $t \rightarrow \infty$. Let $G_{T_{n}}(x)$ be the long-run frequency distribution of the process $\{X(t)\}$ at the imbedded points $T_{n}$, and assume that $G(x)=\lim _{T_{n} \rightarrow \infty} G_{T_{n}}(x)$ exists. Using the relation $Y=\lambda X[1]$, Corollary 5 remains valid when condition $A 1$ is replaced by the following equivalent condition:

$$
\begin{aligned}
\lim _{\alpha \rightarrow \infty} \lim _{n \rightarrow \infty} \frac{1}{n} \sum_{i=1}^{n} \int_{T_{i-1}}^{T_{i}} h(X(u)) \mathbf{1}\{|X(u)|>\alpha\} d u & =0, \\
T_{0} & =0 .
\end{aligned}
$$

Now, we shift attention to stochastic settings and assume that $\{X(t), t \geq 0\}$ is a stochastic regenerative process defined on a probability space $(\Omega, \mathscr{F}, P)$. Let the sequence $\left\{Y_{n}(\alpha)\right\}$, where $Y_{n}(\alpha)=\int_{T_{n-1}}^{T_{n}} h(X(u)) \mathbf{1}\{|X(u)|>\alpha\} d u$, be such that $\left|E Y_{n}(\alpha)\right|=\left|E Y_{1}(\alpha)\right|<\infty$ for all $n=0,1, \ldots$ Then the condition

$$
\lim _{\alpha \rightarrow \infty} E\left(Y_{1}(\alpha)\right)=0
$$

is sufficient for relation (5) to hold with probability one. Note that $\lambda=1 / E T_{1}$ with probability one. Moreover the following result can be easily derived.

Theorem 19. Let $\{X(t), t \geq 0\}$ be a regenerative stochastic process with regeneration points $T_{1}, T_{2}, \ldots$ such that $E\left(T_{1}\right)<$ $\infty$, and $E\left|\int_{0}^{T_{1}} h(X(u)) d u\right|<\infty$; then with probability one

$$
\lim _{t \rightarrow \infty} t^{-1} \int_{0}^{t} h(X(u)) d u=E X_{\infty}
$$

where $X_{\infty}$ has distribution function $G(\cdot)$; that is, relation (5) holds with probability one.

Proof. It follows from the regenerative processes theory, for example, Theorem B.4 in [2], that condition A1 can be written as

$$
\begin{aligned}
& \lim _{\alpha \rightarrow \infty} \lim _{t \rightarrow \infty} t^{-1} \int_{0}^{t} h(X(u)) \mathbf{1}\{|X(u)|>\alpha\} d u \\
& =\lim _{\alpha \rightarrow \infty} \frac{E \int_{0}^{T_{1}} h(X(u)) \mathbf{1}\{|X(u)|>\alpha\} d u}{E\left[T_{1}\right]}=0 .
\end{aligned}
$$

So, we need only to verify that condition (62) is satisfied. Using the dominated convergence theorem [26] and the hypothesis of the theorem, it follows that

$$
\begin{aligned}
& \lim _{\alpha \rightarrow \infty} E\left(Y_{1}(\alpha)\right) \\
& =E \lim _{\alpha \rightarrow \infty} \int_{0}^{T_{1}} h(X(u)) \mathbf{1}\{|X(u)|>\alpha\} d u \\
& =E \int_{0}^{T_{1}} \lim _{\alpha \rightarrow \infty} h(X(u)) \mathbf{1}\{|X(u)|>\alpha\} d u=0 .
\end{aligned}
$$

When $h$ is an identity function, Theorem 19 slightly extends Theorem 11 of Wolff [14, page 92], in the sense that the absolute value of $X(t)$ is not needed in the hypothesis of the theorem.

\section{Competing Interests}

The author declares that they have no competing interests.

\section{Acknowledgments}

The author wishes to thank Professor Shaler Stidham, Jr., for helpful comments, in particular for suggesting Example 15.

\section{References}

[1] S. Stidham Jr. and M. El-Taha, "Sample-path analysis of processes with imbedded point processes," Queueing Systems, vol. 5, no. 1-3, pp. 131-165, 1989.

[2] M. El-Taha and S. Stidham Jr., Sample-Path Analysis of Queueing Systems, Kluwer Academic Publishers, Boston, Mass, USA, 1999.

[3] M. El-Taha, "PASTA and related results," in Wiley EORMS, John Wiley and Sons, New York, NY, USA, 2010.

[4] B. Melamed and W. Whitt, "On arrivals that see time averages," Operations Research, vol. 38, no. 1, pp. 156-172, 1990.

[5] B. Melamed and W. Whitt, "On arrivals that see time averages: a martingale approach," Journal of Applied Probability, vol. 27, no. 2, pp. 376-384, 1990.

[6] R. W. Wolff, "Poisson arrivals see time averages," Operations Research, vol. 30, no. 2, pp. 223-231, 1982.

[7] P. Brémaud, "Characteristics of queueing systems observed at events and the connection between stochastic intensity and palm probability," Queueing Systems, vol. 5, no. 1, pp. 99-111, 1989.

[8] P. Brémaud, R. Kannurpatti, and R. Mazumdar, "Event and time averages: a review," Advances in Applied Probability, vol. 27, pp. 377-411, 1992.

[9] P. Billingsley, Weak Convergence, John Wiley \& Sons, New York, NY, USA, 1968.

[10] R. Serfozo, "Semi-stationary processes," Zeitschrift für Wahrscheinlichkeitstheorie und Verwandte Gebiete, vol. 23, no. 2, pp. 125-132, 1972.

[11] R. F. Serfozo, "Functional limit theorems for stochastic processes based on embedded processes," Advances in Applied Probability, vol. 7, pp. 123-139, 1975.

[12] P. Franken, D. König, U. Arndt, and V. Schmidt, Queues and Point Processes, John Wiley \& Sons, Chichester, UK, 1982.

[13] T. Rolski, Stationary Random Processes Associated with Point Processes, vol. 5 of Lecture Notes in Statistics, Springer, New York, NY, USA, 1981.

[14] R. W. Wolff, Stochastic Modeling and the Theory of Queues, Prentice Hall International Series in Industrial and Systems Engineering, Prentice Hall, Englewood Cliffs, NJ, USA, 1989.

[15] R. Serfozo and S. Stidham Jr., "Semi-stationary clearing processes," Stochastic Processes and Their Applications, vol. 6, no. 2, pp. 165-178, 1978.

[16] J. Stidham Jr., "Regenerative processes in the theory of queues, with applications to the alternating-priority queue," Advances in Applied Probability, vol. 4, pp. 542-577, 1972. 
[17] M. Brown and S. M. Ross, "Asymptotic properties of cumulative processes," SIAM Journal on Applied Mathematics, vol. 22, no. 1, pp. 93-105, 1972.

[18] D. R. Miller, "Existence of limits in regenerative processes," Annals of Mathematical Statistics, vol. 43, pp. 1275-1282, 1972.

[19] H. Toyoizumi, "Sample path analysis of contribution and reward in cooperative groups," Journal of Theoretical Biology, vol. 256, no. 3, pp. 311-314, 2009.

[20] E. Orsingher, C. Ricciuti, and B. Toaldo, "Population models at stochastic times," Advances in Applied Probability, vol. 48, no. 2, pp. 481-498, 2016.

[21] S. Stidham Jr., "A last word on $L=\lambda W$," Operations Research, vol. 22, no. 2, pp. 417-421, 1974.

[22] S. Stidham Jr., "Sample-path analysis of queues," in Applied Probability and Computer Science: The interface, R. Disney and T. Ott, Eds., pp. 41-70, Springer, Berlin, Germany, 1982.

[23] E. Gelenbe, "Stationary deterministic flows in discrete systems. I," Theoretical Computer Science, vol. 23, no. 2, pp. 107-127, 1983.

[24] E. Gelenbe and D. Finkel, "Stationary deterministic flows: II. The single-server queue," Theoretical Computer Science, vol. 52, no. 3, pp. 269-280, 1987.

[25] J.-P. Haddad, R. R. Mazumdar, and F. J. Piera, "Pathwise comparison results for stochastic fluid networks," Queueing Systems: Theory and Applications, vol. 66, no. 2, pp. 155-168, 2010.

[26] P. Billingsley, Probability and Measure, John Wiley \& Sons, New York, NY, USA, 2nd edition, 1985.

[27] K. L. Chung, A Course in Probability Theory, Academic Press, New York, NY, USA, 2nd edition, 1974.

[28] U. Ayesta, "A unifying conservation law for single-server queues," Journal of Applied Probability, vol. 44, no. 4, pp. 10781087, 2007.

[29] D. P. Heyman and M. J. Sobel, Stochastic Models in Operations Research, vol. 1 of McGraw-Hill Series in Quantitative Methods for Management, McGraw-Hill, New York, NY, USA, 1982. 


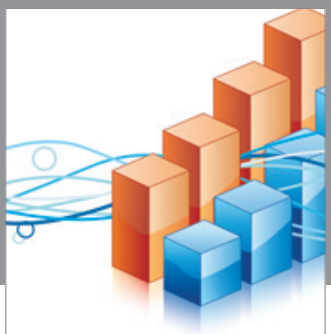

Advances in

Operations Research

vatem alat4

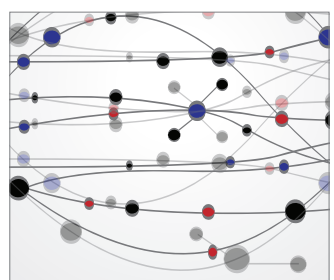

\section{The Scientific} World Journal
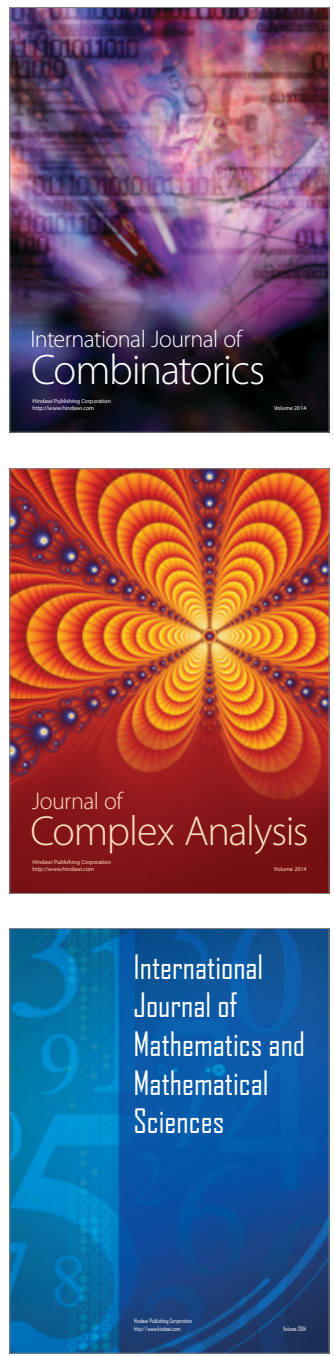
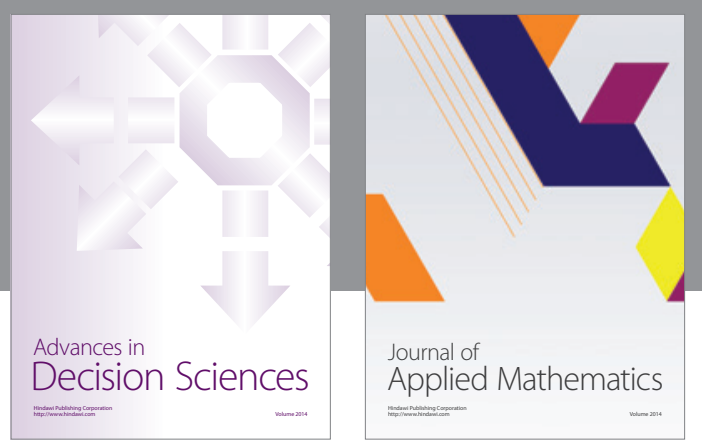

Algebra

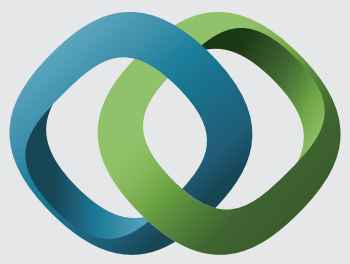

\section{Hindawi}

Submit your manuscripts at

http://www.hindawi.com
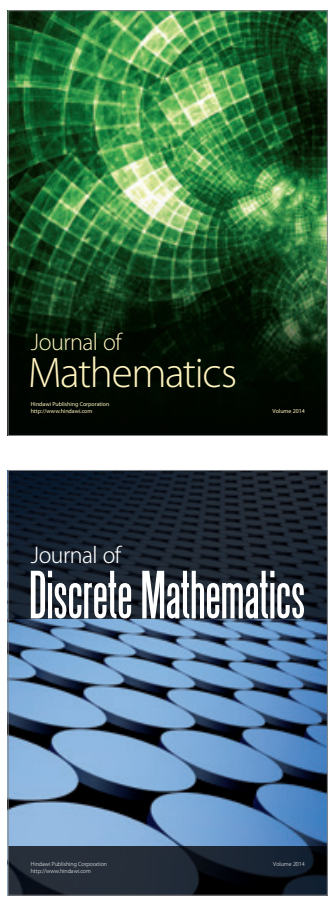

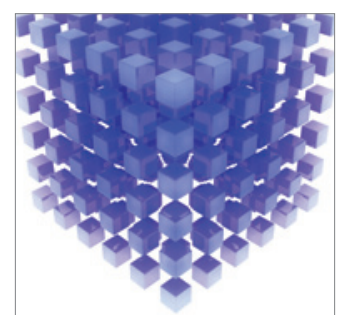

Mathematical Problems in Engineering
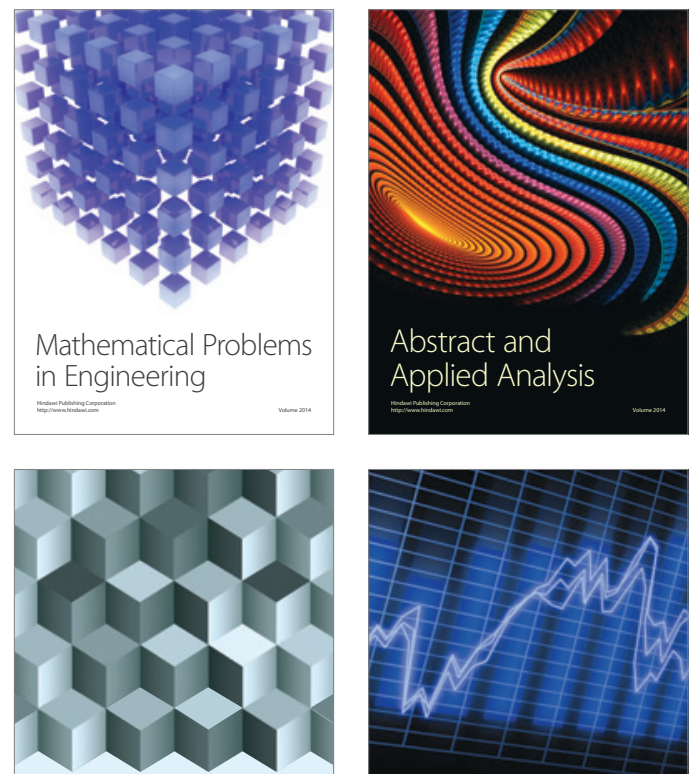

Journal of

Function Spaces

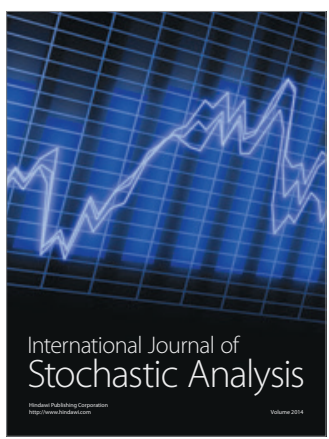

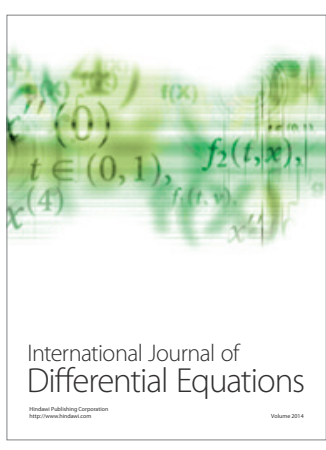
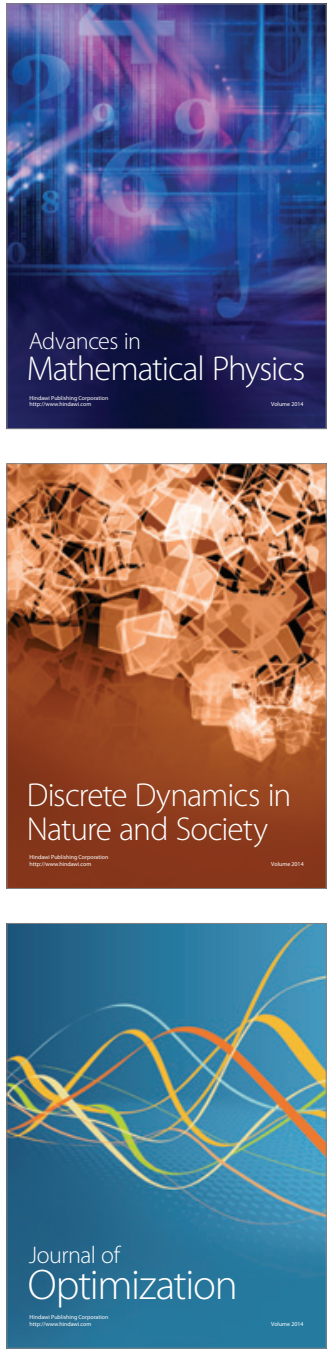